Bull. Austral. Math. Soc.

$08 \mathrm{~A} 55,08 \mathrm{~A} 40$

VOL. 44 (1991) [405-415]

\title{
MINIMAL PARTIAL CLONES
}

\author{
F. Börner, L. Haddad and R. Pöschel
}

\begin{abstract}
Let $A$ be a finite set. A partial clone on $A$ is a composition closed set of operations containing all projections. It is well known that the partial clones on $A$, ordered by inclusion, form a lattice. We show that the minimal partial clones on $A$ are:
\end{abstract}

(a) minimal clones of full operations or

(b) generated by partial projections defined on a totally reflexive and totally symmetric domain.

\section{INTRODUCTION}

Recent developments of universal algebra show the importance of clones as a tool of analysis and classification. A clone is a superposition closed set of finitary operations on a fixed finite set A containing all projections. Maximal clones of total (= everywhere defined) operations are very important for primality (completeness) and are fully known $[10,11]$. Equally minimal clones play a significant role for the following reversed completeness criterion: Given a set $\mathcal{R}$ of relations under which conditions is the clone consisting of all operations preserving all relations in $\mathcal{R}$ reduced to the set of all projections? As the lattice $\mathcal{L}_{O_{A}}$ of all clones is atomic, such a criterion might be based on the knowledge of all operations generating a minimal clone. At the present stage it seems that the determination of minimal clones even for small $|A|$ is a very complex task $[3,6,7,12]$.

Clones of partial operations also play an important role in the theory of partial algebras and in computer science (see for example [2]). Nevertheless they are much less known. Recently maximal partial clones were completely described by combinatorial properties [4]. In the present paper we address the following problem: What are the atoms of the lattice $\mathcal{L}_{P_{A}}$ of all partial clones? We show that they are either the atoms of $\mathcal{L}_{O_{A}}$ or are generated by partial projections defined on a totally reflexive and totally symmetric domain (Theorem 2.5).

Received 20 December 1990

The work has been done during the second author's visit at the Karl-Weierstrass-Institut for Mathematics in Berlin in July 1990. The financial support provided by NSERC Canada is gratefully acknowledged.

Copyright Clearance Centre, Inc. Serial-fee code: 0004-9729/91 \$A2.00+0.00. 


\section{Preliminaries and the main Result}

2.1: Let $A$ be a non-empty set. For a positive integer $n$, an $n$-ary partial operation on $A$ is a map $f: \operatorname{dom} f \rightarrow A$ where $\operatorname{dom} f$ is an $n$-ary relation on $A$ called the domain of $f$. Let $P_{A}^{(n)}$ denote the set of all $n$-ary partial operations on $A$ and let $P_{A}:=\bigcup_{n \geqslant 1} P_{A}^{(n)}$, moreover set

$$
O_{A}^{(n)}:=\left\{f \in P_{A}^{(n)}: \operatorname{dom} f=A^{n}\right\}
$$

and

$$
O_{A}:=\bigcup_{n \geqslant 1} O_{A}^{(n)} \quad \text { (= set of total operations). }
$$

Furthermore for $n \geqslant 1$ we denote by $\operatorname{Rel}_{A}^{(n)}:=\left\{R: R \subseteq A^{n}\right\}$ the set of all $n$-ary relations on $A$ and let $\operatorname{Rel}_{A}:=\bigcup_{n \geqslant 1} \operatorname{Rel}_{A}^{(n)}$. In the sequel we shall distinguish between empty relations of different arities and consequently we shall also distinguish between nowhere defined operations (that is, operations with empty domains) of different arities (see 4.7).

For $C \subseteq P_{A}$ and $n \geqslant 1$, put $C^{(n)}:=C \cap P_{A}^{(n)}$ and let

$$
\mathcal{D}(C):=\{\operatorname{dom} f: f \in C\} .
$$

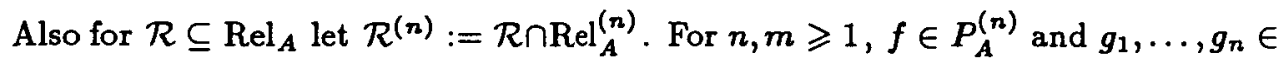
$P_{A}^{(m)}$, we define the superposition of $f$ and $g_{1}, \ldots, g_{n}$, denoted by $f\left[g_{1}, \ldots, g_{n}\right] \in P_{A}^{(m)}$, by setting

$$
\operatorname{dom}\left(f\left[g_{1}, \ldots, g_{n}\right]\right):=\left\{\left(a_{1}, \ldots, a_{m}\right) \in A^{m}:\left(a_{1}, \ldots, a_{m}\right) \in \bigcap_{i=1}^{m} \operatorname{dom} g_{i}\right.
$$

and $\left.\left(g_{1}\left(a_{1}, \ldots, a_{m}\right), \ldots, g_{m}\left(a_{1}, \ldots, a_{m}\right)\right) \in \operatorname{dom} f\right\}$

and

$$
f\left[g_{1}, \ldots, g_{n}\right]\left(a_{1}, \ldots, a_{m}\right):=f\left(g_{1}\left(a_{1}, \ldots, a_{m}\right), \ldots, g_{n}\left(a_{1}, \ldots, a_{m}\right)\right)
$$

for all $\left(a_{1}, \ldots, a_{m}\right) \in \operatorname{dom} f\left[g_{1}, \ldots, g_{n}\right]$.

For every positive integer $n$, every $n$-ary relation $D$ and each $1 \leqslant i \leqslant n$ let $e_{i, D}^{n}$ denote the $n$-ary $i$-th partial projection defined by $e_{i, D}^{n}\left(x_{1}, \ldots, x_{n}\right)=x_{i}$ for all $\left(x_{1}, \ldots, x_{n}\right) \in D$. For notational ease we shall write $e_{i}^{n}$ for $e_{i, A^{n}}^{n}$. Furthermore for $\mathcal{R} \subseteq$ Rel $_{A}$ put

$$
\mathcal{P}(\mathcal{R}):=\left\{e_{i, D}^{n}: 1 \leqslant i \leqslant n<\omega, \quad D \in \mathcal{R}^{(n)}\right\}
$$

hence $\mathcal{P}(\mathcal{R})$ denotes the set of all partial projections with domains in $\mathcal{R}$. Furthermore let

$$
J_{A}:=\left\{e_{i}^{n}: 1 \leqslant i \leqslant n<\omega\right\}
$$


be the set of all total projections.

Definition 2.2: A partial clone on $A$ is a superposition closed subset of $P_{A}$ containing $J_{A}$ (for an equivalent definition see [4]). If a partial clone $C$ contains an $n$ ary operation $f$ with dom $f \neq A^{n}$, then it is called a proper partial clone. If otherwise $C \subseteq O_{A}$ then it is called a total clone. Moreover a partial projection clone is a clone containing only partial projections. For $C \subseteq P_{A}$, let clone $(C)$ denote the partial clone generated by $C$, that is, the least clone containing $C$.

EXAMple 2.3. Let $0 \in A$ and let

$$
\operatorname{Pol}\{0\}:=\bigcup_{n \geqslant 1}\left\{f \in P_{A}^{(n)}:(0, \ldots, 0) \in \operatorname{dom} f \Rightarrow f(0, \ldots, 0)=0\right\},
$$

then $\operatorname{Pol}\{0\}$ is a proper partial clone on $A$.

The partial clones (respectively the total clones) on $A$, ordered by inclusion form an algebraic lattice $\mathcal{L}_{P_{A}}$ [4] (respectively $\mathcal{L}_{O_{A}}$ ) in which every meet is the set-theoretical intersection. For $F \subseteq P_{A}$, the partial clone generated by $F$ is the intersection of all partial clones containing the set $F$ (or equivalently is the set of term operations of the partial algebra $\langle A ; F\rangle)$. A minimal partial clone is an atom of $\mathcal{L}_{P_{A}}$, that is, a partial clone covering the set $J_{A}$. The problem of determining all the atoms of $\mathcal{L}_{O_{A}}$ for $A$ finite was raised in [8], it has been studied by several authors $[3,6,7,12,13]$, and remains unsolved at the present time. However, for $A$ finite, it is known that $\mathcal{L}_{O_{A}}$ is an atomic lattice (that is, every total clone on $A$ distinct from $J_{A}$ contains a minimal total clone) with a finite number of atoms. The five types of minimal total clones are discussed in $[12,13]$.

In order to state our main result we need the following:

2.4. Definition: Let $n \geqslant 1, R$ be an $n$-ary relation on $A$ and $S_{n}$ be the group of permutations on $\underline{n}:=\{1, \ldots, n\}$. The relation $R$ is said to be

(1) totally symmetric if for all $\pi \in S_{n}$ and $\left(a_{1}, \ldots, a_{n}\right) \in A^{n}$,

$$
\left(a_{1}, \ldots, a_{n}\right) \in R \Leftrightarrow\left(a_{\pi(1)}, \ldots, a_{\pi(n)}\right) \in R,
$$

(2) totally reflexive if for every $\left(a_{1}, \ldots, a_{n}\right) \in A^{n}$ and all $1 \leqslant i<j \leqslant n$, the equality $a_{i}=a_{j}$ implies that $\left(a_{1}, \ldots, a_{n}\right) \in R$,

(3) nontrivial if $R \neq A^{n}$.

Note that any subset of $A$ (including the empty set $\emptyset$ ) is considered as a totally reflexive and totally symmetric relation.

Our aim is to show the following result: 
Theorem 2.5. Let $A$ be a finite set with $|A|>1$. The lattice $\mathcal{L}_{P_{A}}$ of all partial clones on $A$ is atomic and contains a finite number of atoms. Moreover $C$ is a minimal partial clone if and only if either $C$ is a minimal total clone or is generated by a single partial projection with a nontrivial totally reflexive and totally symmetric domain.

In the sequel we shall consider a finite set $A$ with $|A|>1$.

The following lemma shows that a minimal proper partial clone is in fact a partial projection clone.

Lемма 2.6. Let $C$ be a partial clone. Then the set $C^{\prime}:=\mathcal{P}(\mathcal{D}(C))$ is a partial projection clone contained in $C$ and such that $\mathcal{D}\left(C^{\prime}\right)=\mathcal{D}(C)$.

Proof: We first show that $C^{\prime} \subseteq C$. Note that $C^{\prime}$ consists only of partial projections. Let $e_{i, D}^{n} \in C^{\prime}$ (for some $1 \leqslant i \leqslant n$ ). Thus $D \in \mathcal{D}(C)$ and consequently there is a $f \in C^{(n)}$ such that $\operatorname{dom} f=D$. As $e_{i, D}^{n}=e_{1}^{2}\left[e_{i}^{n}, f\right]$ we see that $e_{i, D}^{n} \in C$. Now we show that $C^{\prime}$ is a clone. Clearly $J_{A} \subseteq C^{\prime}$ as $\mathcal{D}(C)$ contains all relations $A^{n}, n=1,2, \ldots$ (since $J_{A} \subseteq C$ ). Let $f \in C^{(n)}$ and $g_{1}, \ldots, g_{n} \in C^{(m)}$. It is easy to see that $f\left[g_{1}, \ldots, g_{n}\right]$ is again a partial projection. Moreover $f \in C^{(n)}$ and $g_{1}, \ldots, g_{n} \in C^{(m)}$ because of $C^{\prime} \subseteq C$. As $C$ is a clone, we have that $f\left[g_{1}, \ldots, g_{n}\right] \in C$ and thus $\operatorname{dom}\left(f\left[g_{1}, \ldots, g_{n}\right]\right) \in \mathcal{D}(C)$ proving that $f\left[g_{1}, \ldots, g_{n}\right] \in C^{\prime}$. Thus $C^{\prime}$ is a clone. The equality $\mathcal{D}\left(C^{\prime}\right)=\mathcal{D}(C)$ is obvious.

From Lemma 2.6 we deduce that any partial clone $C$ contains a partial projection clone $C^{\prime}$. If $C$ is a total clone, then $C^{\prime}=J_{A}$ but if $C$ is a proper partial clone, then $C^{\prime}$ contains a not everywhere defined partial projection. Hence we have

Corollary 2.7. Every minimal clone on $A$ is either a total minimal clone (that is, an atom in both $\mathcal{L}_{O_{A}}$ and $\mathcal{L}_{P_{A}}$ ) or is a proper partial projection clone.

Clearly a projection clone $C$ is uniquely determined by the set $\mathcal{D}(C)$ of all the domains of its operations. This leads us to investigate some special sets of relations on $A$ which we call weak systems of relations.

\section{WEAK SYSTEMS OF RELATIONS}

We want to characterise the subsets of $\operatorname{Rel}_{A}$ of the form $\mathcal{D}(C)$ where $C$ is a partial clone on $A$. For this we have to introduce the following concepts:

Definition 3.1: For an integer $n \geqslant 1$ we denote $\underline{n}:=\{1, \ldots, n\}$. Let $n, m \geqslant 1$ be integers and let $s: \underline{n} \rightarrow \underline{m}$ be a map. Define the mapping $t,: \operatorname{Rel}_{A}^{(n)} \rightarrow \operatorname{Rel}_{A}^{(m)}$ by setting

$$
t_{s}(R):=\left\{\left(a_{1}, \ldots, a_{m}\right) \in A^{m}: \quad\left(a_{s(1)}, \ldots, a_{s(n)}\right) \in R\right\}
$$

for all $R \in \operatorname{Rel}_{A}^{(n)}$. 
EXAMPLE 3.2. Let $s: \underline{n} \rightarrow \underline{m}$ be a map.

(1) Let $n=m$ and let $s$ be a permutation on $\underline{n}$. Then $t$, performs a re-arrangement of the arguments of any $R \in \operatorname{Rel}_{A}^{(n)}$. In particular an $n$-ary relation $R$ is totally symmetric if and only if $t_{,}(R)=R$ for all $s \in S_{n}$. Also $R$ is totally reflexive if and only if $t_{r}(R)=A^{n}$ for every non-injective $r: \underline{n} \rightarrow \underline{n}$.

(2) Let $n<m$ and assume $s(k)=k$ for all $k=1, \ldots, n$. Then $t$, adds fictive arguments to any $R \in \operatorname{Rel}_{A}^{(n)}$.

(3) If $s(i)=s(j)$ for some $i \neq j \in \underline{n}$, then $t$, performs the identification of the i-th and j-th argument of any $R \in \operatorname{Rel}_{A}^{(n)}$.

The following results are easy to check:

FACT 3.3. Let $n, m, k \geqslant 1$ be integers, $s: \underline{n} \rightarrow \underline{m}$ and $s^{\prime}: \underline{m} \rightarrow \underline{k}$ be maps and let $R, Q \in \operatorname{Rel}_{A}^{(n)}$. Then

$$
\begin{aligned}
t_{s}\left(A^{n}\right) & =A^{m}, \\
t_{s}(R \cap Q) & =t_{s}(R) \cap t_{s}(Q), \\
t_{s}\left(t_{s}(R)\right) & =t_{s} \operatorname{los}^{\prime}(R)
\end{aligned}
$$

where $\circ$ denotes the composition of maps: $\left(s^{\prime} \circ s\right)(i):=s^{\prime}(s(i))$.

DEFINITION 3.4: A set $\mathcal{R} \subseteq \operatorname{Rel}_{A}$ is said to be a weak system of relations on $A$ if the following conditions hold for all $n, m \geqslant 1$ :

(i) $A^{n} \in \mathcal{R}$,

(ii) for all $R, Q \in \mathcal{R}^{(n)}$ we have $R \cap Q \in \mathcal{R}^{(n)}$ (that is, $\mathcal{R}$ is closed under finite intersections),

(iii) for all $s: \underline{n} \rightarrow \underline{m}$ and all $R \in \mathcal{R}^{(n)}$ we have $t_{s}(R) \in \mathcal{R}^{(m)}$ (that is, $\mathcal{R}$ is closed under all operations $t_{s}$ ).

The following result gives the relationship between partial clones and weak systems of relations:

Proposition 3.5. Let $C$ be a partial clone on $A$. Then $\mathcal{D}(C)$ is a weak system of relations. Conversely if $\mathcal{R}$ is a weak system of relations on $A$ then $\mathcal{P}(\mathcal{R})$ is a partial (projection) clone.

Proof: First let $C$ be a partial clone. We have to show that conditions 3.4 (i) - (iii) hold for $\mathcal{D}(C)$. Let $n, m \geqslant 1$ be integers. As $C$ contains $e_{1}^{n}$, we have $A^{n}=\operatorname{dom} e_{1}^{n} \in \mathcal{D}(C)$. Now let $R, Q \in \mathcal{D}(C)^{(n)}$. Then $R=\operatorname{dom} f$ and $Q=\operatorname{dom} g$ for some $f, g \in C^{(n)}$. Hence $e_{1}^{2}[f, g] \in C^{(n)}$ and $\operatorname{dom}\left(e_{1}^{2}[f, g]\right)=R \cap Q \in \mathcal{D}(C)$. Finally let $s: \underline{n} \rightarrow \underline{m}$ be a map and let $R \in \mathcal{D}(C)^{(n)}$. Again $R=\operatorname{dom} f$ for some $f \in C^{(n)}$. 
Then $h:=f\left[e_{a(1)}^{m}, \ldots, e_{\rho(n)}^{m}\right] \in C^{(m)}$ and hence $\operatorname{dom} h=t_{\bullet}(R) \in \mathcal{D}(C)$. This shows that $\mathcal{D}(C)$ is a weak system of relations. We turn to the proof of the second statement. Let $\mathcal{R}$ be a weak system of relations. We show that $\mathcal{P}(\mathcal{R})$ is a partial clone. Let $n \geqslant 1$ be an integer. From $A^{n} \in \mathcal{R}$ we deduce that $e_{i}^{n} \in \mathcal{P}(\mathcal{R})$ for all $1 \leqslant i \leqslant n$, and thus $J_{A} \subseteq \mathcal{P}(\mathcal{R})$. Now let $n, m \geqslant 1$ be integers, $1 \leqslant j \leqslant n, 1 \leqslant i_{1}, \ldots, i_{n} \leqslant m, Q \in \mathcal{R}^{(n)}$, $R_{1}, \ldots, R_{n} \in \mathcal{R}^{(m)}$ and $f:=e_{j, Q}^{n}, g_{1}:=e_{i_{1}, R_{1}}^{m}, \ldots, g_{n}:=e_{i_{n}, R_{n}}^{m} \in \mathcal{P}(\mathcal{R})$. It is easy to check that $f\left[g_{1}, \ldots, g_{n}\right]=e_{i_{j}, D}^{m}$ where

$$
\begin{aligned}
D & =\left\{\left(x_{1}, \ldots, x_{m}\right) \in \bigcap_{i=1}^{n} R_{i}:\left(x_{i_{1}}, \ldots, x_{i_{n}}\right)=\left(g_{1}\left(x_{1}, \ldots, x_{m}\right), \ldots, g_{n}\left(x_{1}, \ldots, x_{m}\right)\right) \in Q\right\} \\
& =\left(\bigcap_{i=1}^{n} R_{i}\right) \cap t_{\star}(Q)
\end{aligned}
$$

for the map $s: \underline{n} \rightarrow \underline{m}$ defined by $s(k)=i_{k}$.

As $\mathcal{R}$ is a weak system of relations we have that $D \in \mathcal{R}$. This shows that $e_{i_{j}, D}^{m}=$ $f\left[g_{1}, \ldots, g_{n}\right] \in \mathcal{P}(\mathcal{R})$ and completes the proof of Proposition 3.5.

3.6: Denote by $\mathcal{W}_{A}$ the set of all weak systems on $A . \mathcal{W}_{A}$ can be considered as the set of all subalgebras of a many-sorted (heterogeneous) algebra with carrier set $\left(\operatorname{Rel}_{A}^{(n)}\right)_{n \geqslant 1}$ and the operations $A^{n}$ (constants), $\cap$ and $t$, (see 3.4(i)-(iii)). Therefore $\left\langle\mathcal{W}_{A} ; \subseteq\right\rangle$ forms a complete algebraic lattice. The smallest element in this lattice is clearly the weak system

$$
T_{A}:=\left\{A^{n} \mid n \geqslant 1\right\} .
$$

Moreover, given a set $\mathcal{R} \subseteq \operatorname{Rel}_{A}$, the weak system generated by $\mathcal{R}$, which we shall denote by $\langle\mathcal{R}\rangle_{w s}$, is the least weak system containing $\mathcal{R}$. Hence every relation in $\langle\mathcal{R}\rangle_{w}$, can be obtained from $\mathcal{R}$ by applying finitely many times the operations 3.4(i) - (iii) to the relations in $\mathcal{R}$. In particular, if $R \in \operatorname{Rel}_{A}^{(n)}$, then $Q \in\langle R\rangle_{w o}^{(m)}$ (we write $\langle R\rangle_{w}$ s for $\left.\langle\{R\}\rangle_{w s}\right)$ if and only if $Q=f(R)$ for some term operation $f$ build up from the constants $A^{k}(k \geqslant 1)$, the intersections and the operations of the form $t_{\mathrm{s}}$. By applying 3.3 to $f$ as many times as required, we end up with either $Q=A^{m}$ or $Q$ has the following "normal" form $Q=\bigcap_{i=1}^{\ell} t_{s_{i}}(R)$, where $\ell \geqslant 1$ and $s_{1}, \ldots, s_{\ell}: \underline{n} \rightarrow \underline{m}$ are maps. As well-known from universal algebra, $\langle\mathcal{R}\rangle_{w}$, also can be expressed as follows:

$$
\langle\mathcal{R}\rangle_{w_{\bullet}}=\cap\left\{\mathcal{R}^{\prime} \in \mathcal{W}_{A} \mid \mathcal{R} \subseteq \mathcal{R}^{\prime}\right\}
$$

(note that $\mathcal{W}_{A}$ is closed under arbitrary intersections). 
3.7: According to 3.5, the function $\mathcal{D}: C \longmapsto \mathcal{D}(C)$ (respectively $\mathcal{P}: \mathcal{R} \longmapsto \mathcal{P}(\mathcal{R})$ maps $\mathcal{L}_{P_{A}}$ into $\mathcal{W}_{A}$ (resptively $\mathcal{W}_{A}$ into $\mathcal{L}_{P_{A}}$ ). We collect some simple facts: let $\mathcal{R}, \mathcal{R}^{\prime} \subseteq \operatorname{Rel}_{A}, C, C^{\prime} \subseteq P_{A}$, then:

(a) $\mathcal{R}^{\prime} \subset \mathcal{R} \Longrightarrow \mathcal{P}\left(\mathcal{R}^{\prime}\right) \subset \mathcal{P}(\mathcal{R})$,

$C^{\prime} \subseteq C \Longrightarrow \mathcal{D}\left(C^{\prime}\right) \subseteq \mathcal{D}(C)$

$\mathcal{R}=\mathcal{D}(\mathcal{P}(\mathcal{R})), \mathcal{D}(C)=\mathcal{D}(\mathcal{P}(\mathcal{D}(C)))$,

$C \subseteq O_{A} \Longleftrightarrow \mathcal{D}(C) \subseteq T_{A}$.

(b) $C$ is a projection clone $\Longleftrightarrow C=\mathcal{P}\left(\langle\mathcal{D}(C)\rangle_{w s}\right)$.

(c) $\mathcal{R}$ is a weak system $\left(\in \mathcal{W}_{A}\right) \Longleftrightarrow \mathcal{R}=\mathcal{D}$ (clone $\mathcal{P}(\mathcal{R})$ ).

(d) The functions $\mathcal{D}$ and $\mathcal{P}$ provide a monotone (with respect to inclusion) one-to-one correspondence between weak system $\left(\neq T_{A}\right)$ and partial projection clones $\left(\neq J_{A}\right)$. In particular, a proper projection clone $C=\mathcal{P}(\mathcal{R})$ is minimal if and only if $\mathcal{R}=\mathcal{D}(C)$ is a minimal weak system (that is, an atom in $\left.\mathcal{W}_{A}\right)$.

(e) $\langle\mathcal{R})_{w s}=\mathcal{D}($ clone $\mathcal{P}(\mathcal{R}))$.

Proof: (a): follows directly from the definitions.

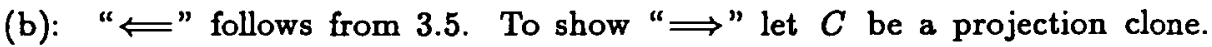
Then obviously $C \subseteq \mathcal{P}(\mathcal{D}(C))$. Now $\mathcal{D}(C)=\langle\mathcal{D}(C)\rangle_{\text {we }}$, by 3.5, and $\mathcal{P}(\mathcal{D}(C)) \subseteq C$ by 2.6 . Thus the equality holds.

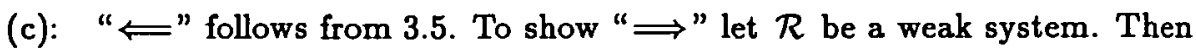
$\mathcal{P}(\mathcal{R})=\operatorname{clone}(\mathcal{P}(\mathcal{R}))$ by 3.5 and we have $\mathcal{R}=\mathcal{D}(\mathcal{P}(\mathcal{R}))=\mathcal{D}$ (clone $\mathcal{P}(\mathcal{R})$ ) (see (a)).

(d): follows from (a), (b), (c) and 3.5.

(e): $\langle\mathcal{R}\rangle_{w}, \subseteq \mathcal{D}$ (clone $\mathcal{P}(\mathcal{R})$ ) follows from (c). Now, $\mathcal{P}(\mathcal{R}) \subseteq \mathcal{P}\left((\mathcal{R}\rangle_{w s}\right)$ implies clone $(\mathcal{P}(\mathcal{R})) \subseteq \mathcal{P}\left((\mathcal{R}\rangle_{\text {ws }}\right)$ (by (b)), thus $\mathcal{D}($ clone $\mathcal{P}(\mathcal{R})$ ) $\subseteq \mathcal{D}\left(\mathcal{P}\left(\langle\mathcal{R}\rangle_{\text {ws }}\right)\right)=\langle\mathcal{R}\rangle_{\text {ws }}$.

\section{Minimal weak Systems of Relations}

Lemma 4.1. Let $\mathcal{R} \in \mathcal{W}_{A}$ and suppose $T_{A} \neq \mathcal{R}$. Then there exists a nontrivial totally reflexive and totally symmetric relation $R \in \mathcal{R}$.

Proof: Put $M:=\left\{n: \mathcal{R}^{(n)} \neq\left\{A^{n}\right\}\right\}$. As $T_{A} \neq \mathcal{R}$ we have that $M \neq \emptyset$. Let $m:=\min M$ and let $Q \in \mathcal{R}^{(m)}, Q \neq A^{m}$. We show that $Q$ is totally reflexive. If $m=1$ then $Q$ is a subset of $A$ and hence is totally reflexive. So assume $m \geqslant 2$. Let $\left(a_{1}, \ldots, a_{m}\right) \in A^{m}$ be such that $a_{i}=a_{j}$ for some $1 \leqslant i<j \leqslant m$. Define $s: \underline{m} \rightarrow \underline{m-1}$ by setting $s(k)=k$ for $k<j, s(j)=i$ and $s(k)=k-1$ for $k>j$. Now by the definition of $m$ we have that $\mathcal{R}^{(m-1)}=\left\{A^{m-1}\right\}$ and as $t,(Q) \in \mathcal{R}^{(m-1)}$ we obtain 
$t_{s}(Q)=A^{m-1}$. In particular $\left(a_{1}, \ldots, a_{j-1}, a_{j+1}, \ldots, a_{m}\right) \in t_{s}(Q)$ and this is possible only if $\left(a_{1}, \ldots, a_{j-1}, a_{i}, a_{j+1}, \ldots, a_{m}\right)=\left(a_{1}, \ldots, a_{m}\right) \in Q$. Thus $Q$ is totally reflexive. Next put

$$
R:=\cap\left\{t_{s}(Q): s \in S_{m}\right\}
$$

By $3.2(1)$ and 3.3, $R$ is totally reflexive and totally symmetric. As $Q \in \mathcal{R} \in \mathcal{W}_{A}$ we have that $R \in \mathcal{R}$. Furthermore from $Q \neq A^{m}$ we get $R \in A^{m}$.

We now describe all minimal weak systems of relations on $A$ :

Lемма 4.2. Let $n \geqslant 1$ and $R \in \operatorname{Rel}_{A}^{(n)}$ be nontrivial, totally reflexive and totally symmetric. Then $\langle R\rangle_{w}$, is a minimal weak system of relations on $A$, that is $\langle R\rangle_{w s}$ is an atom of $\left\langle\mathcal{W}_{A} ; \subseteq\right\rangle$.

Proof: Let $\mathcal{R} \in \mathcal{W}_{A}$ be such that $T_{A} \neq \mathcal{R} \subseteq\langle R\rangle$. We show that $R \in \mathcal{R}$. As $\mathcal{R} \neq T_{A}$, there is an $m \geqslant 1$ and a $Q \in \mathcal{R}^{(m)}$ such that $Q \neq A^{m}$. Since $Q \in\langle R\rangle$, we have

$$
Q=\bigcap_{i=1}^{\ell} t_{s i}(R)
$$

by 3.6 , where $\ell \geqslant 1$ and $s_{1}, \ldots, s_{\ell}: \underline{n} \rightarrow \underline{m}$ are maps. If some $s_{i}$ is non-injective $(1 \leqslant i \leqslant \ell)$, then we get $t_{s_{i}}(R)=A^{m}$ (because $R$ is totally reflexive, see $3.2(1)$ ) and as $Q \neq A^{m}$ we have that $s_{i}$ is injective for at least one $i \in\{1, \ldots, \ell\}$, say $s_{1}$. Let $u: \underline{m} \rightarrow \underline{n}$ be a map such that the composition $u \circ s_{1}$ is the identity map id on $\underline{n}(i d(k)=k$ for $k \in \underline{n})$. Now by $\left(^{*}\right)$

$$
t_{u}(Q)=t_{u}\left(\bigcap_{i=1}^{\ell} t_{s i_{i}}(R)\right)
$$

and from 3.3 we have

$$
t_{u}(Q)=t_{i d}(R) \cap\left(\bigcap_{i=2}^{\ell} t_{u \Omega_{i} i}(R)\right)
$$

Clearly $t_{i d}(R)=R$. Also note that $t_{u \circ s_{i}}(R)=A^{n}$ if $u \circ s_{i}: \underline{n} \rightarrow \underline{n}$ is not injective and $t_{u \circ o_{i}}(R)=R$ if $u \circ s_{i}$ is injective (see 3.2(1)). Thus $R=t_{u}(Q) \in \mathcal{R}$. This shows that $\langle R\rangle_{w,}=\mathcal{R}$ and completes the proof of the Lemma.

REMARK 4.3: Let $n, m \geqslant 1, R \in \operatorname{Rel}_{A}^{(n)}, Q \in \operatorname{Rel}_{A}^{(m)}$ be two nontrivial totally reflexive and totally symmetric relations. Then the equality $\langle R\rangle_{w}=\langle Q\rangle_{w}$ implies that $n=m$ and $R=Q$. Indeed from the proof above we get that $R=t_{u}(Q)$ and $Q=t_{v}(R)$ for some maps $u: \underline{m} \rightarrow \underline{n}, v: \underline{n} \rightarrow \underline{m}$. As both $R$ and $Q$ are nontrivial and totally reflexive we have that both $u$ and $v$ are injective (see 3.2(1)). Hence $n=m$ and $u, v \in S_{n}$. Moreover from $R$ and $Q$ totally symmetric we deduce that $R=Q$. 
We collect the above results $(4.1,4.2,3.7(\mathrm{~d}))$ to obtain

Corollary 4.4. Let $A$ be a finite set and $C$ be a proper partial clone on $A$. Then $C$ is a minimal proper partial clone if and only if $\mathcal{P}(C)$ is a minimal weak system of relations whence if and only if $C$ is generated by a proper partial projection whose domain is a nontrivial totally reflexive and totally symmetric relation.

4.5: We are now in a position to prove Theorem 2.5:

We first show that the lattice $\mathcal{L}_{P_{A}}$ is atomic. Let $C$ be a partial clone. If $C$ is a total clone then it contains a minimal total clone since $\mathcal{L}_{O_{A}}$ is atomic (see for example, $[8,3.1 .5])$. Otherwise $C$ is a proper partial clone. Thus $C$ contains $\mathcal{P}(\mathcal{D}(C)$ ) (by 2.6), and $\mathcal{D}(C)$ contains a minimal weak system $\mathcal{R}$ by 4.1 and 4.2. Therefore $C$ contains the minimal partial clone $\mathcal{P}(\mathcal{R})$ (see $3.7(\mathrm{~d})$ ). Hence $\mathcal{L}_{P_{\mathcal{A}}}$ is atomic.

As there are only finitely many minimal total clones (see for example $[13,1.14,8$, 12]) and finitely many non-trivial totally reflexive and totally symmetric relations on $A$ (their arities are bounded by $|A|$ ), we deduce (see 2.7 and 4.4 ) that the number of minimal partial clones on $A$ is finite. The remaining part of Theorem 2.5 is Corollary 4.4 .

Let $|A|=k>1$ and denote by $\tau(k)$ the number of minimal total clones on $A$ (that is $\tau(k)$ is the number of atoms of $\mathcal{L}_{O_{A}}$ and this number is unknown for $k>3$ ). Using 2.5, 4.4 and 4.3 we get

Corollary 4.6. The number $m(k)$ of minimal partial clones on $A$ with $|A|=$ $k \geqslant 2$ is

$$
m(k)=\tau(k)+\sum_{\ell=1}^{k}\left(2^{\left(\begin{array}{l}
k \\
\ell
\end{array}\right)}-1\right) .
$$

In particular we have $\tau(2)=7, m(2)=11, \tau(3)=84, m(3)=99$.

ProOF: By 4.3 and 4.4, we have to count all different totally reflexive and totally symmetric relations on $A$. Let $1 \leqslant \ell \leqslant k$ and $R \in \operatorname{Rel}_{A}^{(\ell)}$ be totally reflexive and totally symmetric. Thus $R$ contains the relation

$$
R_{0}:=\left\{\left(a_{1}, \ldots, a_{\ell}\right) \in A^{\ell}:\left|\left\{a_{1}, \ldots, a_{\ell}\right\}\right|<\ell\right\}
$$

and moreover $\left(a_{1}, \ldots, a_{\ell}\right) \in R$ implies $\left(a_{\pi(1)}, \ldots, a_{\pi(\ell)}\right) \in R$ for all $\pi \in S_{\ell}$. Consequently either $R=R_{0}$ or

$$
R=R_{0} \cup \bigcup_{i=1}^{0}\left\{\left(a_{\pi(1)}^{i}, \ldots, a_{\pi(\ell)}^{i}\right): \pi \in S_{\ell}\right\}
$$

for some $\ell$-element subsets $A_{i}=\left\{a_{1}^{i}, \ldots, a_{\ell}^{i}\right\} \subset A, i \in\{1, \ldots, s\}$, where $1 \leqslant s \leqslant\left(\begin{array}{l}k \\ \ell\end{array}\right)$.

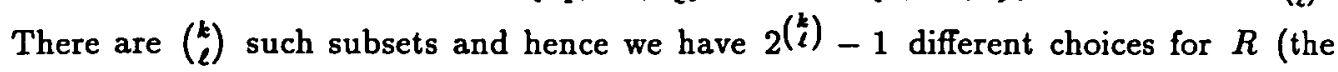


-1 comes from the fact that $R=A^{\ell}$ is excluded, $R=R_{0}$ corresponds to the choice of no subset $A_{i}$ ). This establishes the formula for $m(k)$. Now it is well known that $\tau(2)=7, \tau(3)=84[9,3]$.

REMARKS 4.7: (1) For every $n \geqslant 1$ denote by $o_{n}$ the $n$-ary partial operation on $A$ with empty domain; that is dom $o_{n}=\theta_{n}$ (where $\theta_{n} \in \operatorname{Rel}_{A}^{(n)}$ denotes the empty set considered as the empty $n$-ary relation). Then every $\theta_{n}$ generates the weak system

$$
\left\langle\emptyset_{1}\right\rangle_{w \bullet}=\left\langle\emptyset_{n}\right\rangle_{w \bullet}=T_{A} \cup\left\{\emptyset_{n}: n \geqslant 1\right\}
$$

(since $t_{s}\left(\emptyset_{n}\right)=\emptyset_{m}$ for $s: \underline{n} \rightarrow \underline{m}$ ) which is minimal since $\emptyset_{1} \in \operatorname{Rel}_{A}^{(1)}$ is totally reflexive and totally symmetric. The corresponding minimal partial clone is

$$
\mathcal{P}\left(\left\langle\emptyset_{1}\right\rangle_{w \bullet}\right)=J_{A} \cup\left\{o_{n} \mid n \geqslant 1\right\}
$$

This clone actually corresponds to $\ell=1$ and $R=\emptyset_{1}$ in the counting above.

(2) In [5] partial clones are defined differently and one can show that our definition and the definition given in [5] are not equivalent (every partial clone in the sense of 2.2 is a clone in the sense of [5] but not conversely). The problem of describing all minimal partial clones according to the definition in [6] is still open.

\section{REFERENCES}

[1] F. Börner, Operationen auf Relationen, Dissertation (Universität Leipzig, 1988).

[2] P. Burmeister, A model theoretic oriented approach to partial algebras (Introduction to theory and applications of partial algebras Part I) 32 (Math. Research, Akademie Verlag Berlin, 1986).

[3] B. Csákány, 'All minimal clones on the three-element set', Acta Cybernet. 6 (1983), 227-238.

[4] L. Haddad and I.G. Rosenberg, 'Completeness theory for finite partial algebras', Algebra Universalis (to appear).

[5] L. Haddad, I.G. Rosenberg and D. Schweigert, 'A maximal partial clone and a Slupecki type criterion', Acta. Sci. Math. 54 (1990), 89-98.

[6] P.P. Pálfy, 'Minimal clones', preprint 27/1984, Math. Inst. Hungarian Acad. Sci. (1984).

[7] P.P. Pálfy, The arity of minimal clones, Acta Sci. Math. 50 (1986), 331-333.

[8] R. Pöschel and L.A. Kalužnin, Funktionen und Relationenalgebren. Ein Kapitel der diskreten Mathematik (Deutscher Verlag der Wiss., Berlin, 1979 and Birkhauser, Basel, Stuttgart 1979).

[9] E.L. Post, 'The two-valued iterative systems of mathematical logic', Ann. Math. Studies 5 (1941).

[10] I.G. Rosenberg, 'La structure des fonctions de plusieurs variables sur un ensemble fini', C.R. Acad. Sci. Paris Sér. A-B 260 (1965), 3817-3819. 
[11] I.G. Rosenberg, 'Über die funktionale Vollständigkeit in dem mehrwertigen Logiken', Rozpravy Cs. Akademie Vëd. Ser. Math. Nat. Sci. 80 (1970), 3-93.

[12] I.G. Rosenberg, 'Minimal clones I: The five types', in Lectures in Universal Algebra 43, Colloq. Math. Soc. J. Bolyai, pp. 405-427, 1983.

[13] A. Szendrei, Clones in universal algebra 99, Séminaire de Mathématiques Supérieures (Université de Montréal, Canada, 1986).

Karl-Weierstrass-Institut for Mathematics Mohrenstrasse 39

Berlin 1086

Germany

Karl-Weierstrass-Institut for Mathematics Mohrenstrasse 39

Berlin 1086

Germany
Department of Mathematics University of Toronto Toronto, Ontario Canada M5S 1A1 